\title{
Frontières
}

\section{Olivier Larronde ou la marche à la mort}

\section{Lorca Hassan Almohammed}

Volume 19, numéro 2, printemps 2007

\section{Penser sa mort?}

URI : https://id.erudit.org/iderudit/017496ar

DOI : https://doi.org/10.7202/017496ar

Aller au sommaire du numéro

Éditeur(s)

Université du Québec à Montréal

ISSN

1180-3479 (imprimé)

1916-0976 (numérique)

Découvrir la revue

\section{Citer cet article}

Almohammed, L. (2007). Olivier Larronde ou la marche à la mort. Frontières, 19(2), 41-46. https://doi.org/10.7202/017496ar

\section{Résumé de l'article}

La pensée de la mort inspire au poète français Olivier Larronde des images dynamiques. Ces images, particulièrement lugubres, s'inscrivent à la fois dans la vie et dans l'oeuvre du poète, suscitant une véritable marche à la mort (d'où l'intitulé de l'article). Dans l'écriture, les pensées et les images de la mort contribuent à une mise en scène, celle de la fin macabre du poète. D’un poème à l'autre, la mort est transcrite sous forme poétique. L'univers poétique de la pensée lugubre reflète, d'une part, l'analyse des images de la mort et, de l'autre, dévoile cette marche à la mort devenue l'écho intérieur de l'imaginaire matériel. Cet imaginaire est le miroitement d'une conscience foncièrement mélancolique qui traverse les poèmes de Larronde.
Ce document est protégé par la loi sur le droit d'auteur. L'utilisation des services d'Érudit (y compris la reproduction) est assujettie à sa politique d'utilisation que vous pouvez consulter en ligne.

https://apropos.erudit.org/fr/usagers/politique-dutilisation/ 


\section{Résumé}

La pensée de la mort inspire au poète français Olivier Larronde des images dynamiques. Ces images, particulièrement lugubres, s'inscrivent à la fois dans la vie et dans l'œuvre du poète, suscitant une véritable marche à la mort (d'où l'intitulé de l'article). Dans l'écriture, les pensées et les images de la mort contribuent à une mise en scène, celle de la fin macabre du poète. D'un poème à l'autre, la mort est transcrite sous forme poétique. L'univers poétique de la pensée lugubre reflète, d'une part, l'analyse des images de la mort et, de l'autre, dévoile cette marche à la mort devenue l'écho intérieur de l'imaginaire matériel. Cet imaginaire est le miroitement d'une conscience foncièrement mélancolique qui traverse les poèmes de Larronde.

Mots clés: Poésie - mort - pensée image.

\section{Abstract}

This article is a consideration of the dynamic images of death in the work of French poet Olivier Larronde. Singularly gloomy images figure simultaneously in his life and work and give rise to a true "march to death". In his writing, the thoughts and images inspired by death help set the stage for the end of his own life. From one poem to the next, death is transcribed in poetic form. The poetic universe of this thinking reflects an analysis of the images of death and reveals the march to death as the internal echo of his imaginings. This imaginative world is a projection of the fundamentally melancholy consciousness that runs through Larronde's poems.

Keywords: Poetry - Death - Thought Image.

\section{OLIVIER LARRONDE OU LA MARCHE À LA MORT}

\section{Lorca Hassan Almohammed, doctorant au Centre de recherches sur les littératures modernes et contemporaines, Université Blaise-Pascal, France.}

Nous proposons ici une lecture des images poétiques interchangeables qui marquent l'évolution de la pensée de la mort chez Olivier Larronde ${ }^{1}$. L'analyse des images de la mort dans son œuvre permet d'en distinguer deux types. L'«image imaginante» (Bachelard, 2004) et l'«image pensante » ou l'«être pensant» (Jankélévitch, 1977). Cette distinction montrera que la philosophie de la mort n'est pas étrangère à l'imaginaire de la mort. Aussi voudrions-nous explorer l'écriture poétique de la mort même du poète. Nous proposerons donc une réflexion sur la poétique du moi vis-à-vis de la mort propre dans la poésie de Larronde en tant que marche consciente à la mort.

Dans La Mort, Jankélévitch dit: «Tout me parle de la mort mais indirectement et à mots couverts, par hiéroglyphes et sousentendus. La vie est l'épiphanie de la mort, mais cette épiphanie est allégorique, non point tautégorique; ce sont des allusions qu'il faut savoir comprendre » (1977, p. 59). Il est clair que la prémonition de la mort repose sur des allusions allégoriques dans l'univers poétique d'Olivier Larronde. Nous examinerons les dimensions et les

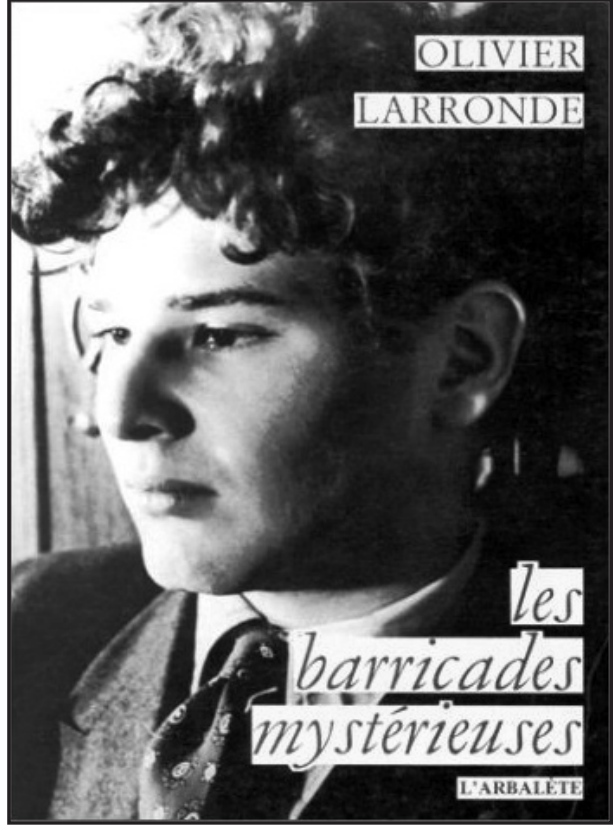

limites de la problématique prémonitoire de la mort chez ce poète pour avoir accès à son monde intérieur, dans lequel les images de la mort propre donnent lieu à une pensée de la mort apprivoisée. Il faut souligner cependant un parallélisme entre la pensée de la mort et son image qui s'impose au cœur de la problématique de l'écriture poétique mortifère, d'où la prédominance des métaphores relatives à la mort. 


\section{Éléments biographiques}

- 1927, naissance d'Olivier Iris Hélios Larronde à La Ciotat en France.

- Fin 1939, fin d'une enfance idyllique.

- 1942, Olivier Larronde fut placé dans un collège religieux à Bury, non loin de Saint-Leu-la-Forêt.

- 1943, il décide de monter à Paris pour y rencontrer Jean Cocteau. Celui-ci lui déconseillera de poursuivre dans la voie poétique.

C'est Jean Genet qui lui ouvrit les bras.

- La même année, première édition des Barricades mystérieuses illustrées par André Beaurepaire. Max-Pol Fouchet publiera ce recueil dans la revue Fontaine. Sartre en publie quelques poèmes dans Les Temps modernes.

- 1947-1948, première attaque de «haut mal»

- 1959, Marc Barbezat publie Rien Voilà l'ordre aux éditions L'Arbalète, illustré de trente et un dessins d'Alberto Giacometti.

- 1965, mort d'Olivier Larronde à Paris. II repose à côté de la tombe de Mallarmé.

- 1966, publication de son troisième recueil L'Arbre à Lettres aux éditions L'Arbalète.

Néanmoins il serait intéressant de voir jusqu'où l'image de la mort peut refléter la pensée de la mort dans l'écriture poétique; autrement dit, comment l'image de la mort est susceptible de devenir une pensée de la mort. Nul doute que la lecture de l'œuvre poétique d'Olivier Larronde nous engage dans une "philosophie imaginaire» de la pensée morbide, car elle nous conduit à la constitution d'éléments caractérisant l'expérience intérieure de la mort propre dans son évolution métaphorique et imaginaire. Tout en étant dissociées, l'intellection de la mort et l'imagination de l'espace de la mort sont convergentes parce qu'elles aboutissent à de multiples significations dans le contexte métaphorique de la mort. C'est-à-dire que chaque image implicite ou explicite de la mort semble alimenter une pensée de la mort du poète.

Chez nombre de jeunes poètes hantés par leur mort, la lecture spatiotemporelle des images prémonitoires de la mort repose sur trois éléments. D'abord métaphorique, l'image de la mort ne va pas au-delà de son aspect emblématique. L'autre élément relève d'un ordre métaphysique qui se traduit par l'obsession du poète pour sa propre mort, obsession qui tend à se transformer en une pensée de la mort. Le dernier élément appartient au registre poétique, en ce sens où la mort, en tant qu'image et en tant que pensée, se fixe dans le poème et ne cesse de traverser l'âme du poète. Olivier Larronde, quant à lui, écrit, décrit et imagine sa mort, car il la pressent. Chez lui, la poétique du moi dénote une pensée de la mort qui est analogue à une marche à la mort. Dans les dernières années de son existence, Larronde était conscient de l'approche de sa mort. Dans sa poésie, l'imagination de la mort passe de la simple évocation (métaphorique) à la pensée de la mort (métaphysique), de la présence textuelle de l'image de la mort à sa conceptualisation. D'où l'on peut conclure à l'existence de l'imaginaire prémonitoire de la mort propre, qui correspond à la jonction de l'image de la mort avec la pensée de la mort. En effet, sens et image ne sont jamais séparés chez Larronde, puisque leur fusion constitue la métaphore prémonitoire de la mort, c'est-à-dire la métaphore consciente de la mort du poète. Les barricades mystérieuses ${ }^{2}$ intensifient justement le sens et l'image de la vie indépassable et incompréhensible. Elles constituent l'emblème de l'expérience intérieure de l'angoisse et de la mort chez le filleul de Milosz ${ }^{3}$.

Puisque « la vie parle de la mort» comme le résume bien Jankélévitch, la vie - faisant basculer l'être mais aussi basculée par l'être - devient une source de mort qui peut s'exprimer dans deux modes d'existence complètement contradictoires. D'une part, la confrontation du poète à un monde extérieur homogène, qui le condamne à un état d'enfermement subversif et funeste. D'autre part, le monde intérieur hétérogène se définissant comme une réaction à l'étrangeté du monde extérieur dans lequel le poète est contraint de vivre. Incontestablement ce monde-là ne lui appartient plus car c'est un monde qui lui arrache son univers intérieur. Larronde n'est plus attiré par le monde des hommes. Il est à la recherche d'un monde qui n'existe pas. Conscient de la vanité de sa quête d'un monde digne de lui, Larronde tente d'oublier les deux mondes - intérieur et extérieur - à la fois. Le monde extérieur l'effraie en ce qu'il a de superficiel, d'éphémère, de mensonger et surtout d'incompréhensible. C'est pourquoi il va se livrer totalement à la claustrophobie et à sa condition mélancolique. Le repli sur soi est donc inévitable, il ne lui reste plus rien à faire dans un monde qui lui devient insupportable, à l'instar de Danielle Collobert ${ }^{4}$ qui n'arrivait plus à supporter la vie. On peut donc aisément comprendre pourquoi Larronde se plongeait à l'excès dans l'alcool et se laissait consumer par l'opium. C'est ici qu'apparaît la marche lente et inéluctable à la mort. Désormais, Larronde semble vivre dans un monde de souvenirs irrécupérables. Pour autant le monde intérieur du poète n'est que la transformation du monde extérieur. L'appel du plaisir, des sens, des couleurs et des parfums retrouvés dans un monde imaginaire et mémoriel s'avère funeste avec le temps. Tout lui devient étouffant, absurde et ennuyeux. Car les souvenirs d'enfance et d'amours sont d'abord attachés à son passé. Les limites sensorielles et mémorielles définitives du monde fixent le monde larrondien seulement dans la mémoire du poète. Par conséquent, tout le pousse à la dérision. L'auteur des Barricades mystérieuses s'invente un monde dans lequel il s'emprisonne. Le temps même n'est plus supportable. C'est alors le pressentiment de la perte de soi :

\section{Instant Je pétris tu écorches \\ Jaloux de ta forme informe \\ Là! je t'élance ...et moi m'effeuille... \\ Ma terre en boue Très loin Brille. \\ (2004, p. 98)}

Le monde devient en soi une barricade qui isole complètement le poète de la réalité extérieure. Une marche à sa «terre en boue » qui «Très loin / Brille », s'avère être aussi une marche à une rêverie solitaire. Tout ce qui se rapporte à la mort du poète est énoncé sur le mode ironique et mélancolique. Son désir de tisser un monde qui lui ressemble devient le signe même de son désespoir. Même la tentative de récupérer l'espoir de vivre s'annonce comme une tentative de réconciliation irréalisable avec la vie. Seul le monde idyllique est à même de faire émerger le poète du cercle maléfique, malfaisant du monde extérieur.

Allée de peupliers (1884), Vincent Van Gogh. 


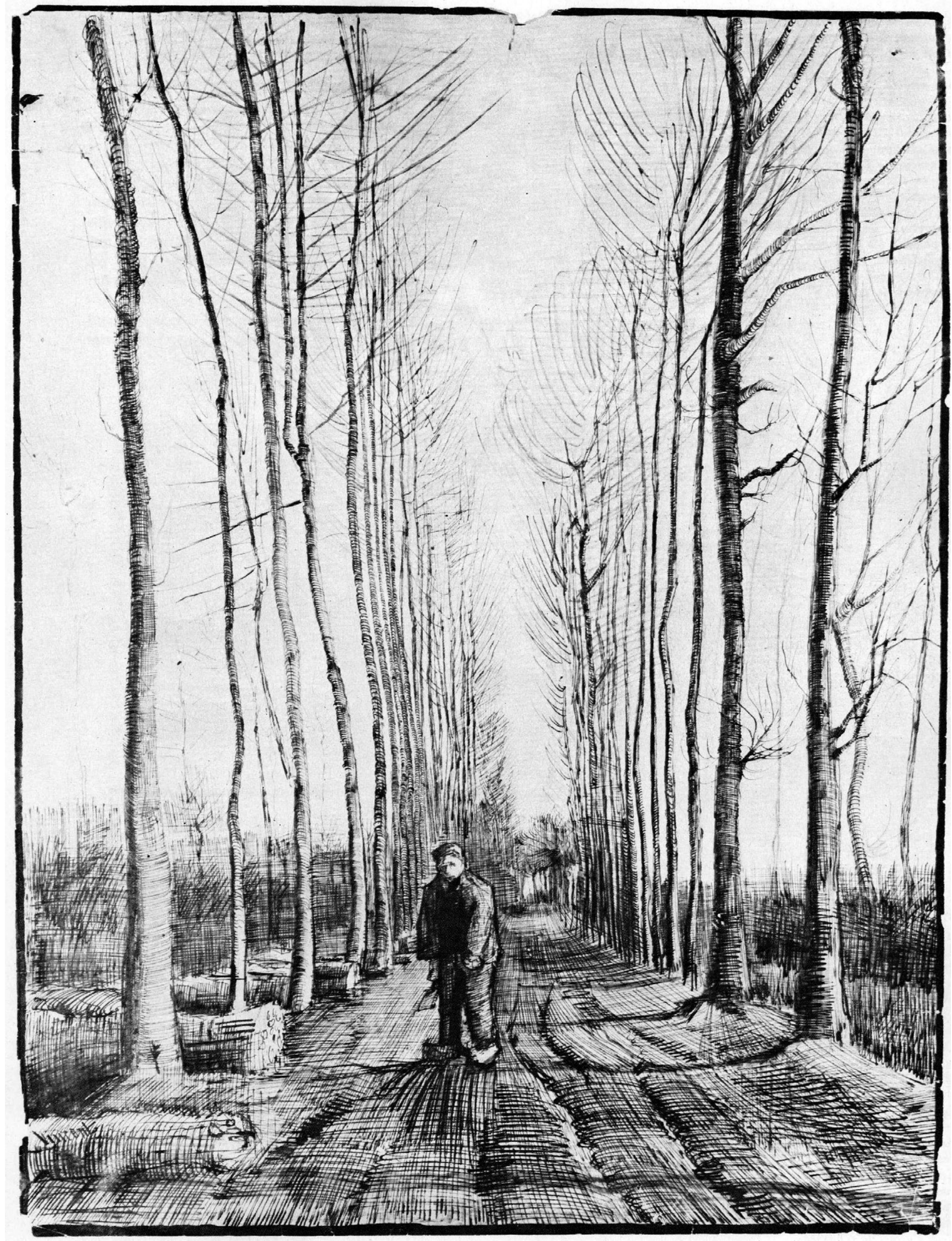


Or l'univers intérieur se heurte au monde réel et le poète est condamné à se délester de ses illusions. Il s'opère alors « un choc», à la fois extérieur et intérieur. Le monde de fleurs et d'oiseaux se lie à un «monde poétique» qui lui sert de langage autre lui permettant de tromper la mort, de jouer avec elle, d'escamoter mélancolie et douleur, d'occuper pleinement son temps dans l'espoir de fuir un monde ennuyeux.

Cependant cette évasion s'avère en fait impossible: «le Spleen haï ${ }^{5}$ » de Larronde est présent partout. Il est caché dans le sens même de l'écriture et se définit à la conjoncture de l'absurde et de l'angoisse. Le monde imaginaire et poétique ne sera donc qu'un refuge dans un microcosme de papier qui lui servira de lieu légendaire de prédilection. Un lieu vital d'abri et de protection contre le temps qui le harcèle. Mais Larronde est parfaitement conscient du fait qu'il se constitue de la sorte un refuge chimérique et éphémère. Il se réfugie alors dans la nature qui, paradoxalement, va contribuer à ses déchirements et accroître ses tourments. La nature, barricade qui le nourrit d'une grande détresse, s'avère finalement hostile à son égard. Tout ce qui précède fournit donc les éléments premiers de l'expérience intérieure dont témoignent Les barricades mystérieuses.

Pour ce qui est du sens poétique, la pesanteur ontologique des Barricades mystérieuses se compose, selon nous, de deux éléments fondamentaux ayant pour origine l'angoisse permanente d'une marche à la mort dans l'œuvre d'Olivier Larronde. Ces deux éléments se rattachent au monde mystérieux du poète. Il s'agit de l'ennui et de l'absurde. L'ennui donne libre cours au despotisme de l'idée de l'absurde et de la pensée morbide. Tout se banalise à partir du moment où l'expérience vitale et poétique de l'ennui se transforme, de temps à autre, en une quête de fondements substantiels de l'existence. La simultanéité de l'expérience existentielle peut se traduire par des questions transcrites dans le poème, «im-posées» au poète. Larronde ne va pas pouvoir trouver de réponses à ces questions, qu'il se contente de (se) poser. La question demeure posée pour lui et fournit par ellemême une réponse. La question qui hante l'expérience intérieure de Larronde porte sur la mort. Les barricades mystérieuses suggèrent une marche occulte à la mort, ne fût-ce que pour ériger un obstacle face au monde imaginaire du poète. Même impensable, la métaphysique de la mort devient de ce fait imaginable, voire envisageable. Afin de voir les diverses connotations médiates de la mort, nous proposons une lecture d'une métaphore corporelle, dynamique et vitale inhérente à la mort. Il s'agit de l'image $\mathrm{du}$ « ramoneur».
Rare et curieuse, la métaphore du « ramoneur » suggère une véritable marche à la mort chez Larronde, car elle incarne une autoconsommation de soi. Cette métaphore va montrer l'évolution du sens caché de la pensée mortifère. C'est une image exploratrice et dynamique; elle est d'abord là pour faire allusion à l'imagination «méta-physique» du poète. La marche et les pas du «ramoneur» font référence à la fatigue et à la lassitude face à la vie. Cependant les idées inférées par l'image obsessionnelle du «ramoneur» dépassent le simple aspect emblématique, venant nuancer l'évolution imaginaire de l'état intérieur du poète. Cette image signifie l'ennui, la monotonie, l'angoisse et l'absurde. Tels sont les degrés consécutifs du travail imaginaire $d u$ "ramoneur».

La métaphore du «ramoneur»-adressée à l'autre - fait partie des «fonctions qui résistent à la mort ${ }^{6} »$ (Xavier Bichat, p. 1). Néanmoins elle devient funeste et létale, car elle tient lieu d'une pensée morbide inévitable du poète. Larronde ne livre pas une simple métaphore d'une «image imaginante» (Bachelard, 2004). Au contraire, il donne libre cours à l'imagination de sa mort progressive qui peut se traduire justement par la rencontre de l'«image imaginante» et de l'«image pensante» (Jankélévitch, 1977). La fusion de ces deux natures d'image n'est d'ailleurs pas facile à cerner. Mais elle permet de former les linéaments de telle ou telle autre image de la mort. Sans doute la métaphore du «ramoneur» est-elle un rappel de l'ennui dans l'œuvre de Larronde. La comparaison du poète avec le «ramoneur» donne lieu à des images qui chargent les poèmes larrondiens d'un nouveau souffle. Cette comparaison met l'accent sur l'inextricable parcours poétique d'une symbolique qui oppose le noir au blanc, la vie à la mort dans l'espace parcouru des poèmes, celui de la vérité poétique incernable, c'est-à-dire de la liberté insaisissable. Le «ramoneur» est en quelque sorte une image "sisyphique» qui relève d'une intensité poétique sans égale chez Larronde. C'est une réflexion essentielle et immédiate sur l'absurde. Jean-Pierre Lacloche, ami très proche du poète, le confirme : Il [Larronde] opposa - s'engagea dans un face-à-face féroce avec l'absurde ${ }^{7}$. » La métaphore en question est l'incarnation parfaite de l'absurde et de l'ennui chez Larronde comme étant source de la mort. Le «ramoneur» est aussi la figuration d'un état d'errance existentielle. Le «ramoneur» renvoie métaphoriquement à l'angoisse et à la noirceur. Le travail $\mathrm{du}$ « ramoneur» met en relief l'incarnation même de la fatuité de la vie et de son ennui funeste. Le poète tente en vain de se débarrasser de cette figure du «ramoneur» qui le côtoie. C'est donc une marche «parasuicidaire». Le besoin de mettre fin à l'angoisse ainsi qu'à l'ennui devient lancinant. Larronde s'apprête à embrasser la mort dans ses textes, qui témoignent d'une sorte de culpabilité de vivre et d'exister. Pour se rendre à la terre, le poète va devoir se désapproprier de ses impulsions de «ramoneur». Larronde retrouve son salut provisoire, celui de l'écriture. Écoutons-le:

Qu'à tes gestes, beau nègre,

on accroche une rose,

En serais-tu vexé? Tu la gifles, elle pleure

Comme aux gifles du vent

je perdrais mes couleurs,

Elle aussi : ta peau de ramoneur est en cause

(2002, p. 38).

Le «ramoneur » représente une humeur caractérielle du personnage poétique. Il y a un lien étroit entre cette «image imaginante» (Bachelard, 2004) des "gifles du vent», qui démontre parfaitement l'existence perturbée du poète. "Les gifles du vent» sont celles de la vie touchant à sa fin. La suite du troisième vers montre bien cette inclination de perte de soi chez le poète d'«Ivraie en ronde»: «je perdrais mes couleurs », couleurs qui font référence au personnage sentimentaliste et même pathétique du poète perdant la vie, parce que la rejetant. On est donc face à une confrontation de la vie et de la mort. Larronde est un poète qui procède parfois par l'entremise d'une algèbre intérieure. Il est happé par cette quête de nombres en parallèle avec les lettres. Il y a là de quoi s'interroger sur le langage adaptable à la hantise de la mort pressentie. Plus le thème s'avère embrouillé, plus le langage s'embrouille et s'approche du langage des nombres. Cette algèbre poétique, le poète s'en sert pour faire l'équilibre entre la vie et la mort et pour fuir l'ennui. Un tel langage est en quelque sorte nihiliste. Il met les mots hors le sens des mots, c'est-à-dire qu'il impose le nombre, masqué parfois par les lettres au profit du sens des mots. C'est un recours, par le langage algébrique, à un rajout de la mort à l'affût même de l'écriture puisque celle-ci représente aussi la vie du poète confrontée à la mort, sinon déjà à elle préparée. Le « ramoneur» identifie en ce sens le fond imprononçable du poète. Il incarne ainsi une expression identifiable. Le "ramoneur» est avant tout «un», signalant l'unicité du poète menacée par la mort. Il serait donc juste de dire aussi que la mort habite l'écriture malgré l'unicité bouleversée et partagée entre le poète et la mort. Dans les années 1947 et 1948, Olivier Larronde subit sa première crise de haut mal. Sans doute 
faudrait-il y ajouter la hantise du mal-être. Car, après tout, c'est cette habitation du mal-être projeté dans l'œuvre de Larronde qui donne naissance, d'une manière ou d'une autre, à cette coulée de noir dégagée par la densité des images comme celle du «ramoneur». Dans la poésie de Larronde, la tâche du «ramoneur» consiste donc à rejeter l'absurde et à fuir l'ennui, donc la vie. Le mouvement provoqué par le dynamisme du «ramoneur» est une interrogation sur l'attente de la mort et de l'Inconnu. C'est enfin, et par métaphore, une gageure importante compte tenu du caractère «para-suicidaire» du poète qui se refuse à se situer à mi-chemin de l'au-delà, à se placer corps et âme dans une vie tenue pour morte. Pour lui, il n'y a pas de juste milieu. Il demeure à cheval sur ses désirs de triompher de la mort par la mort même. On est donc face à une sorte de réconciliation mystérieuse entre le un et son double, entre le poète et sa mort.

À la croisée des rapprochements des rapports au mystère chez Larronde, la métaphore dynamique du «ramoneur» s'identifie à l'idée de la mort dans la vie. On pourrait y voir un état de conflit et une condition d'anxiété qui se traduisent par une communication étrange et troublée avec le monde des choses. Arrivant à saturation, le poète se trouve en deçà du seuil infranchissable des choses. Dès lors, il est condamné à vivre dans le tumulte du ramonage inespéré. Ces allées et venues du mouvement exercé par le "ramoneur» vont conduire par leur mécanisme à un questionnement sur la condition absurde et monotone d'une vie extrêmement ennuyeuse. Cela porte par là même une interrogation sur la "fatigabilité » à l'égard du simple exercice répétitif qu'est la vie. Cette "fatigabilité» semble être due dans sa totalité à l'existence même. Le ressassement est donc en butte à la maladresse, autre figure du mouvement. De son vivant, le poète fut un maladroit. La cassure des choses fait penser à l'auto-cassure de soi. La maladresse de Larronde habite ses textes: les vitres, le verre, les corps métalliques cassés ou déformés sont présents dans son œuvre. Parallèlement, on est devant une harmonie entre l'objet et le mot. La cassure et le brisement sont des termes importants pour Larronde. Mais la cassure des choses rappelle surtout la provocation de la mort ou la mort provocatrice. La mort serait par métaphore quelque chose qui casse maladroitement la vie du poète. Il y a dans l'univers poétique de Larronde un balancement à la fois vital, communicatif et linguistique établi avec le monde incompréhensible et flou des choses. Ce balancement peut signifier à quelques endroits près une défaite face à la mort. Il s'agit d'une mort à la fois apprivoisée, appréhendée et paradoxalement assumée par le poète. Ce balancement de vie et de mort se revêt derechef d'un arrièreplan noir. La mort est ce noir qui habite la vie; par ailleurs la vie est page blanche. Cependant, le balancement d'idées fléchit du côté du noir.

Dans la poésie de Larronde, il est rarissime de trouver l'évocation d'une peinture grise qui puisse rappeler ce mélange entre vie et mort au milieu duquel se situe le poète météore ${ }^{8}$. Tout comme la lumière opposée à l'obscurité, le noir s'oppose souvent au blanc chez Larronde. Plus curieux encore, la référence à l'image de la pluie représente l'opposition de la vie à la mort, car malgré sa préfiguration d'a priori de la vie, la pluie s'avère être un élément de destruction, de corrosion et de dévastation du corps de la terre. Ciel et terre sont deux éléments poétiques de la vie et de la mort dans l'univers de Larronde. Ainsi, dans sa pensée poétique, la pluie se traduit-elle par la complicité du Mal dans la nature associée au mal-être du poète. Il y a donc lieu de parler d'une discontinuité qui s'impose dans l'écriture poétique de Larronde. Le partage d'amour avec la nature et la régression de l'amour pour la nature dérivent justement de cette discontinuité. Toutefois l'attitude de Larronde à l'égard de la nature n'est pas sans importance en dépit des brouilles discontinues avec la nature et ses éléments :

Je me dispute avec le soir fragile et casse

Casse comme une vitre

et j'ai plusieurs cadavres

On me recueille, on me recolle, et on se lasse:

Je couche avec un coin de mur que mon aire navre

(2002, p. 38)

Il s'agit d'une discontinuité d'idées, laquelle rappelle sans doute celle du « ramoneur» qui gratte en vain de la suie, du noir, ce que Larronde considère comme étant le plus haut mal. Ainsi le ravalement des murs noirs dans l'image pensante du «ramoneur »est métaphorisé par l'écriture comme acte d'insistance sur la volonté de survivre. Cependant, l'écriture se métamorphose dans une sorte de lecture quasiment gnostique. C'est dire que le poète retranscrit sa méditation et lui donne chair sous forme de mots. Il s'agit d'inscrire le ressentiment incompris de la mort dans le texte. Dorénavant, c'est l'association des images et des métaphores évocatrices de la mort qui donnent à réfléchir et à prédire les fragments flous et révélateurs du sort du poète. Et c'est à travers la pensée poétique de la poésie prémonitoire de Larronde que le sort du poète se dévoile. Faisant partie de l'univers prémonitoire, la nature contribue, elle aussi, à la surabondance d'ennui. Ainsi la pluie est l'expression d'une contradiction et d'une menace pour l'existence humaine. La pluie se personnifie pour exprimer l'état de déstabilisation et d'effroi du poète :

La pluie montre ses dents, exige la lumière

Mon envie de crier, comme un doigt qu'on déplie,

Tire, tire les fils du nez

de la mercière

Qui maigrit, mais qui tourne, embobinant la pluie

(2002, p. 38).

Le caractère mélancolique de Larronde s'identifie bien aux choses en mouvement. Le poète se familiarise avec les choses qui lui ressemblent. En ce sens, le «soir fragile» cité dans le premier quatrain rappelle la fragilité de Larronde. Dans le deuxième quatrain, le retour aux éléments de la nature vient mettre l'accent sur cette mélancolie fatale qui caractérise le poète. L'envie de crier signale un mouvement de recul et de défaillance devant la pluie dont la chute plus ou moins verticale semble être à l'origine de l'harmonisation avec le dépliement du doigt. Il s'agit de mouvements architecturaux qui répondent, du même coup, à deux figurations spatiotemporelles dans le texte. La verticalité se croise avec l'horizontalité. Le point de croisement donne à mystifier les choses. La verticalité peut désigner un mouvement temporel qui fait référence au ciel, symbole de la mort. L'horizontalité dont le poète veut se débarrasser désigne plutôt l'espace dans lequel Larronde se sent emprisonné. Cet espace est à double sens : d'abord l'espace du monde intérieur, ensuite celui du monde extérieur, à savoir la terre. Verticalité et horizontalité veulent dire intérieur et extérieur, ciel et terre, et donc vie et mort. À regarder le mécanisme de l'image $d u$ «ramoneur», on pourrait aisément imaginer un ensemble de mouvements mécaniques qui, dans la pure imagination, semblent vouloir éradiquer l'espace noir à la fois du vu et du vécu. La métaphore du «ramoneur» n'est pas une simple métaphore qui embellirait la poésie de Larronde. Tout au contraire, elle s'avère être l'outil poétique par excellence pour lutter contre le chaos qui afflige le poète. Le «ramoneur» est justement là comme tentative d'effacer ce point de croisement - qui est une autre barricade infranchissable - du mouvement vertical avec le mouvement horizontal. Là aussi, ces deux mouvements répétitivement croisés renvoient à la présence du «sisyphique » et de 
l'ennui. Comme pour l'image dynamique du forgeron commentée par Bachelard (2004, p. 131-174), le «ramoneur» est là pour démystifier les barricades tenues pour indépassables. Seule la mort lui permettrait de franchir ces barricades. Sans nul doute la mort est-elle pour Larronde le meilleur moyen d'esquiver cet écueil qui consiste à sortir une fois pour toutes de cette algèbre noire de la vie, sortir de soi à la façon de Jean-Pierre Duprey ${ }^{9}$. En fait, Larronde préfère la mort progressive, comme une bombe à retardement, le suicide par soumission totale et excessive à l'alcool. Il choisit le liquide comme moyen d'annihiler la vie. L'univers aquatique dans l'œuvre de Larronde n'est pas sans importance. L'eau, se transformant en substance corrosive capable de ronger corps et terre, manifeste l'envie de quitter la terre et de se débarrasser du corps, en l'occurrence, celui du poète.

C'est ainsi que Les barricades mystérieuses vont s'imprégner d'une expérience intérieure de la mort, une expérience biologique, physiologique et bucolique attisée par l'ennui intérieur, incommunicable à l'autre, introspectif et profondément existentiel. C'est un ennui inexorable ancré dans l'âme même du poète, un ennui angoissant étroitement lié à l'expérience originelle de la vie. L'ennui que provoquent les mouvements répétés du "ramoneur» se présente comme l'expression de l'angoisse en permanence, l'ennui qui dévorait Sylvia Plath ${ }^{10}$. Il va donc falloir au poète démolir toutes les barricades temporelles. Mais Larronde aura préféré tuer le temps comme Danielle Collobert. C'est le temps et l'ennui qui mangent la vie du poète.

Enfin le paradoxe entre la pensée et l'image, entre la philosophie et la poésie peut se justifier quand le poète a recours à sa faculté de penser. Comment penser sa mort? Jankélévitch (1977) nous apprend que la mort est impensable; il est possible cependant de penser que la mort est imaginable à la manière du poète. Car en poésie tout peut devenir pensée et image à la fois. Tel est le paradoxe premier de la pensée de la mort imaginaire/imaginable. L'expérience prémonitoire de la mort apprend donc que la poésie prémonitoire est d'abord prophétique. Elle livre une clé au poète déchiré par la vie. Elle lui apprend à mourir. C'est une poésie qui annonce la mort du poète. Larronde voit dans la poésie non seulement une prophétie mais aussi cette douleur qu'elle lui procure: "C'était écrit au fil d'hiéroglyphes / Poésie reconnaissons ta griffe.»Et plus loin: Sans nom sans ouïe sans rien qu'on aime, / Née pour griffer dedans, / Son poète» (2002, "Chat-shacha», p. 153). Ainsi, et comme c'est le cas pour beaucoup de poètes dont la vie a été brève, la poésie peut être le mode d'écriture à part entière en ce que ce mode a d'allusif et d'immédiat. À la différence de plusieurs autres genres littéraires, la poésie de Larronde est prémonitoire dans la mesure où elle laisse prévoir des horizons ignorés et des zones nouvelles de la géographie poétique du tréfonds du poète. Elle apprend à dire, à apprivoiser et à pressentir la mort dans la profondeur de l'homme poète. Mais il faut prendre en compte le contexte du poème, c'est-à-dire savoir à quel moment le poème a été écrit dans la vie du poète et dans quelles circonstances. Il n'est donc pas toujours possible de cerner le contexte du poème au moment de son écriture. C'est pourquoi une lecture à multiples facettes doit être privilégiée. L'expérience intérieure de la mort chez Olivier Larronde est fondée sur de réelles allusions. C'est une expérience retardatrice de la mort. Le poète est donc capable de pressentir et d'imaginer sa mort quand il se sent chassé du paradis terrestre. Dès le début, Olivier Larronde pressentait sa propre mort «Continuer la vie autour d'un mort: / c'est moi [...] » (2002, «L'écorce», p. 191).

\section{Bibliographie}

BACHELARD, G. (2004). La Terre et les rêveries de la volonté, Paris, Librairie José Corti.

BICHAT, M.F.X. (non daté) Recherches physiologiques sur la vie et la mort, Paris, Chez Besson, Gabon et Cie, Libraires, rue PierreSarrazin, $n^{\circ} 7$, et près de l'École de Médecine, $\mathrm{n}^{\circ}$ 33. An VIII.

JANKÉLÉVITCH, V. (1977). La Mort, Paris, Flammarion.

LARRONDE, O. (2002). EEuvres poétiques complètes, Paris, Éditions Gallimard, Coll. «Le promeneur».

\section{Notes}

1. Grand poète français tombé aujourd'hui dans l'oubli. Né en 1927 à La Ciotat, mort le jour des Morts en 1965, à Paris.

2. Ce premier recueil du poète figure dans Olivier Larronde, Euvres poétiques complètes, les Éditions Gallimard, le Promeneur, Paris, 2002. C'est cette même édition que j'adopte ici.

3. En effet, Larronde fut le filleul de Milosz qui se lia d'amitié avec le père de Larronde, Carlos Larronde, lui aussi poète.

4. Poète née à Rostrenen en 1940 ; elle s'est suicidée en juillet 1978 , le jour de son anniversaire. Danielle Collobert s'inscrit dans la même lignée des jeunes poètes météores dont la marche poétique et vitale fut une marche métaphorique à la mort.

5. Terme utilisé par l'ami du poète Jean-Pierre Lacloche dans sa préface des Euvres poétiques complètes d'Olivier Larronde.

6. Terme utilisé pour faire allusion à Xavier Bichet pour qui «la vie est l'ensemble des fonctions qui résistent à la mort».

7. Témoignage de Jean-Pierre Laroche, cité dans Larronde, 2002.

8. J'entends par «météore» le poète ayant vécu une brève vie durant laquelle il brille et s'éteint précocement.

9. Jean-Pierre Duprey (1930-1959), autre poète de brève vie qui mettait l'accent sur la pensée suicidaire dans ses poèmes.

10. Autre grande figure de la poésie, Sylvia Plath inscrivit, elle aussi, sa propre marche à la mort qui se rapproche de celle de Larronde. 